\title{
Fructose-Induced Cognitive Dysfunction Is Associated with Increased Oxidative Stress in the Rat Brains
}

\author{
En Yabe' ${ }^{1}$, Shoko Yamakawa ${ }^{1}$, Hiroe Sakuyama Tomari1 ${ }^{1}$, Yuri Kintaka², Yoshio Uehara1 ${ }^{*}$ \\ ${ }^{1}$ Division of Clinical Nutrition, Faculty of Home Economics, Kyoritsu Women's University, Tokyo, Japan \\ ${ }^{2}$ Division of Health and Nutrition, Jumonji University, Niiza, Japan \\ Email: *yuehara@kyoritsu-wu.ac.jp
}

How to cite this paper: Yabe, E., Yamakawa, S., Tomari, H.S., Kintaka, Y. and Uehara, Y. (2018) Fructose-Induced Cognitive Dysfunction Is Associated with Increased Oxidative Stress in the Rat Brains. Journal of Biosciences and Medicines, 6, 52-64. https://doi.org/10.4236/jbm.2018.610007

Received: September 20, 2018

Accepted: October 23, 2018

Published: October 26, 2018

Copyright $\odot 2018$ by authors and Scientific Research Publishing Inc. This work is licensed under the Creative Commons Attribution International License (CC BY 4.0).

http://creativecommons.org/licenses/by/4.0/

\begin{abstract}
High fructose consumption is a risk factor for diabetes. Type 2 diabetes is associated with cognitive and memory impairment. To determine if fructose intake might affect memory function independently of diabetes development, we investigated the effects of monosaccharides on memory in prediabetic state. We examined whether $2.5 \%$ apple juice solution could influence cognitive function in salt-sensitive Dahl rats using passive avoidance methods. Furthermore, we examined the role of monosaccharides intake in cognitive function and oxidative stress in the brain. Four-week consumption of either apple juice or $2.5 \%$ fructose did not affect the blood glucose concentrations; however, apple juice, but not fructose, significantly decreased cognitive function compared with that of control rats given water. Second, Wistar rats aged 4 weeks were assigned to four groups given water, or $1.25 \%$ glucose, or fructose, or galactose solutions for 11 weeks. The fructose group had only slightly higher blood glucose concentrations than the control group. However, memory function engraved at age of 7 weeks and evaluated for 8 weeks in a passive avoidance test, was significantly decreased in the fructose and galactose groups compared with those in the glucose group. Oxidative stress in the brain, assessed by the tissue malondialdehyde (MDA) content, was significantly increased in the fructose group compared with that in the control group, and the decrease in cognitive function significantly correlated with the MDA content. These findings suggest that long-term apple juice consumption decreases memory function, possibly through an increase in oxidative stress in the brain, in turn induced by fructose overloading. Fructose-induced cognitive dysfunction is likely mediated by mechanisms other than insulin resistance.
\end{abstract}




\section{Keywords}

Fructose, Cognitive Function, Type 2 Diabetes, Dahl Rat

\section{Introduction}

According to National Health and Nutrition Surveillance in Japan, the prevalence of diabetes has been increasing over the decades [1]. More than $90 \%$ of Japanese patients have type 2 diabetes, whereas Caucasian people in the US and Europe commonly suffer from type 1 diabetes. The reason for the high prevalence of type 2 diabetes in Japan is not clear; however, genetic background, life style, and food culture may contribute to the regional differences.

Recent studies have reported a role of excessive fructose intake in the onset of type 2 diabetes in rodents and humans [2] [3] [4] [5] [6]. Elderly people in Japan generally consume at least $150 \mathrm{~g}$ of sweet fruits per day [7]. Based on a fructose content of $15^{\circ} \mathrm{Bx}$, this amount translates to an average daily fructose intake of as much as $22 \mathrm{~g}$. In US, averaged fructose intake reaches $48 \mathrm{~g}$ (37\% of all sugar), and such high fructose intake is a potential risk factor for type 2 diabetes [8]. Diabetes is a main cause of renal impairment and end-stage renal disease requiring hemodialysis or kidney transplants. Proteinuria and insulin glycosylation or hypersecretion play critical roles in diabetic renal impairment. Therefore, fructose intake management is important to reduce the risk of obesity and diabetes in Japan.

Evidence is emerging that obesity and type 2 diabetes are often associated with cognitive impairment [9] [10] [11] [12] [13]. With the elderly population growing, the number of potentially affected individuals has been increasing. However, it is not clear whether cognitive dysfunction is a consequence of diabetes. Favoring causation are reports of impaired glucose utilization, decreased insulin sensitivity in the central nervous system and increased glycosylation in hippocampal cells responsible for memory in diabetic patients [10] [14]. Furthermore, hyperinsulinemia has been shown to accelerate the processes of neural aging and neurodegeneration [12]. In addition, evidence exists that fructose-induced diabetes is associated with impaired cognition, likely through the disruption of the blood-brain barrier (BBB) [15] [16]. The BBB is susceptible to oxidative stress; therefore, fructose-related cognitive impairment may be mediated by an increase in oxidative stress in the brain.

Given that fructose increases oxidative stress on its own, it is important to distinguish clinically between cognitive impairment resulting from diabetes and that associated with excessive intake of fructose per se. To this end, in the present study, we tested the hypothesis that low-dose fructose intake might be associated with cognitive impairment in prediabetes. Further, we examined the role of cerebral oxidative stress caused by excessive fructose consumption in cognitive impairment in rats. 


\section{Materials and Methods}

\subsection{Experimental Design}

\subsubsection{Experiment I. Effects of Apple Juice on Cognitive Function}

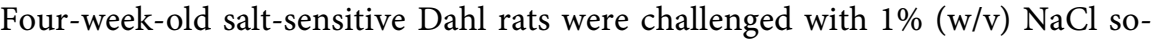
lution for 3 weeks (Sankyo Laboratory, Tokyo, Japan) to increase the blood pressure, and then randomly assigned to 1) a control group given water or 2) a group given $2.5^{\circ} \mathrm{Bx}$ straight apple juice (approximately 6-fold diluted commercially available pure juice). The rats were maintained on water or apple juice for 4 weeks. Regular 0.6\% (w/w) NaCl chow (Oriental Kobo Co., Ltd.), and drinking water or juice solution were available ad libitum during the experiment.

Per $100 \mathrm{~mL}$, the $2.5^{\circ} \mathrm{Bx}$ apple juice given to the rats contained $33 \mathrm{mg}$ of protein, $16 \mathrm{mg}$ of lipid, $2.0 \mathrm{~g}$ of carbohydrate, $0.5 \mathrm{mg}$ of $\mathrm{Na}^{+}, 12.8 \mathrm{mg}$ of $\mathrm{K}^{+}, 0.3 \mathrm{mg}$ of $\mathrm{Ca}^{2+}$, and $0.5 \mathrm{mg}$ of $\mathrm{Mg}^{2+}$, with a total energy value of $7.3 \mathrm{kcal}$. The regular chow used in the present study was made according to the American Institute of Nutrition recommendations for animal research (AIN-76 and AIN-93). For every $100 \mathrm{~g}$, the chow mix contained $23.8 \mathrm{~g}$ of protein, $5.1 \mathrm{~g}$ of lipid, $54.0 \mathrm{~g}$ of carbohydrate, $3.2 \mathrm{~g}$ of fiber, $0.24 \mathrm{~g}$ of $\mathrm{Na}^{+}, 0.87 \mathrm{~g}$ of $\mathrm{K}^{+}$, and $1.11 \mathrm{~g}$ of $\mathrm{Ca}^{2+}$, with other micronutrients and vitamins, and a total energy value of $357 \mathrm{kcal}$. Each solution and the chow were available ad libitum for 11 weeks.

At the end of the experiment, each rat was placed in a metabolic cage and a 24 $\mathrm{hr}$ urine sample was collected [17] [18]. After $12 \mathrm{hr}$ fasting, the rats were anesthetized with pentobarbital $(75 \mathrm{mg} / \mathrm{kg}$ body weight [BW]), and blood samples and the organs of interest were obtained. The samples were stored under $-80^{\circ} \mathrm{C}$ until the assay.

\subsubsection{Experiment II. Effects of Long-Term Intake of Monosaccharides on Cognitive Function}

Wistar male rats aged 4 weeks were purchased from Sankyo Laboratory. The rats were fed a regular chow diet (Oriental Kobo). At the age of 7 weeks, the rats were randomly assigned to 1) a control group given water, 2) a group given $1.25 \%(w / v)$ glucose solution (Wako Pure Chemicals, Tokyo, Japan), 3) a group given $1.25 \%(\mathrm{w} / \mathrm{v})$ fructose solution (Wako Pure Chemicals), or 4$)$ a group given $1.25 \%(\mathrm{w} / \mathrm{v})$ galactose solution (Wako Pure Chemicals).

At the end of the experiment, each rat was placed in a metabolic cage and a 24 hr urine sample was collected [17] [18]. After $12 \mathrm{hr}$ fasting, the rats were anesthetized with pentobarbital (75 mg/kg body weight [BW]), and blood samples and the organs of interest were obtained. The samples were stored under $-80^{\circ} \mathrm{C}$ until the assay.

\subsection{Biochemical and Blood Pressure Measurements}

The systolic blood pressure was determined by the tail-cuff method (Natsume Manometer-Tachometer model KN-210-1, Tokyo, Japan) [17] [18]. Protein excretion in the urine was determined using the Protein assay kit (BioRad, Tokyo, Japan). Blood and urine electrolytes were determined by an autoanalyzer. Blood 
glucose concentrations were determined by the glucose oxidase method.

\subsection{Evaluation of Learning Ability by the Passive Avoidance Task}

The behavioral experiments were performed in a quiet, diffusely lighted room (indirect light from 25-Watt lamps placed $1.5 \mathrm{~m}$ above the animals; O'HARA \& CO., Ltd., Tokyo, Japan) [19] [20] [21]. After the rats had acclimated to the test room, they were trained in a conventional step-through passive avoidance apparatus that was divided into two chambers, one light and the other dark (Figure 1). The apparatus had a stainless-steel grid floor, and the chambers were separated by a sliding door. Each animal was placed initially in the safe lighted chamber with the slit door closed. After $60 \mathrm{~s}$ of equilibrium stabilization, the slit door to the dark chamber was opened. After the rat stepped into the dark room, the door was closed and an electric foot shock (75 V, $500 \mathrm{~mA}$; parameters determined according to previous studies [19]) was delivered. After $10 \mathrm{~s}$, the door was re-opened and the rat regained access to the safe light room. Through a series of such procedures, the rats memorized the risk of a foot shock in the dark compartment and its avoidance in the light one.

To determine how well the memory was preserved, response latency was measured. Briefly, the shock generator was turned off and the rats were placed in the safe light chamber. The time to enter the dark chamber was measured, for a maximum of $400 \mathrm{~s}$. Longer response latencies indicated better memory function.

\subsection{Evaluation of Oxidative Stress in the Brain}

To assess oxidative stress in the brain, we measured malondialdehyde (MDA)

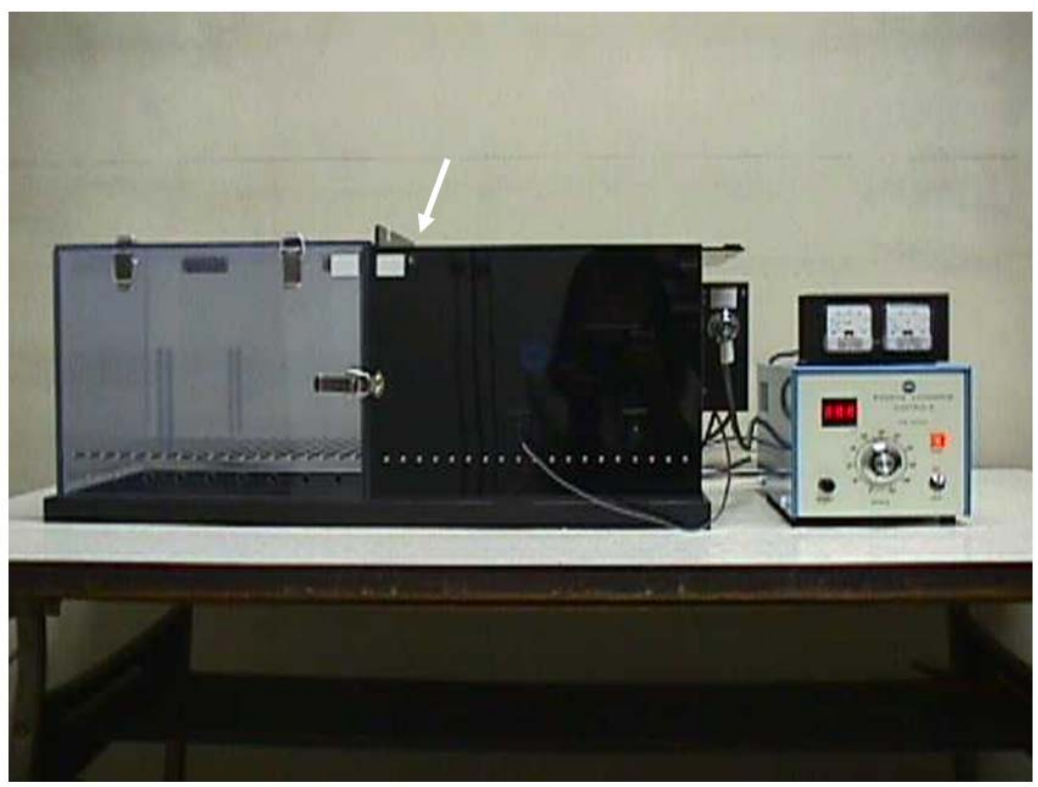

Figure 1. Passive avoidance apparatus. The apparatus had two chambers, light room (left) and dark room (right), separated by a slit door indicated by a white arrow. The right device is a controller to place an electric shock to engrave the memory as detailed in text. The latency time more than 400 secs was considered as maintaining the memory. 
generation in whole-brain homogenates [22] [23]. Briefly, the entire brain was isolated, rinsed with ice-chilled fresh Dulbecco's phosphate-buffered solution (D-PBS), and quickly homogenized in 50 mmoles/L D-PBS, pH 7.4, at $4^{\circ} \mathrm{C}$ using a Polytron ultrasonic blender (Central Scientific Commerce, Inc., Tokyo, Japan). The homogenate was spun at $1300 \mathrm{~g}$ at $4^{\circ} \mathrm{C}$ for $10 \mathrm{~min}$. An aliquot of the supernatant was incubated at $37^{\circ} \mathrm{C}$ for $30 \mathrm{~min}$, and the reaction was terminated with $20 \%$ trichloroacetic acid $(1: 1, \mathrm{v} / \mathrm{v})$. The assay mixture was immediately centrifuged at $2000 \mathrm{~g}$ at $4^{\circ} \mathrm{C}$ for $15 \mathrm{~min}$. Two $\mathrm{mL}$ of the supernatant was heat-treated at $100^{\circ} \mathrm{C}$ for $15 \mathrm{~min}$ with $0.4 \mathrm{~mL}$ of $0.12 \mathrm{M}$ thiobarbituric acid solution, $\mathrm{pH}$ 7.4. The optical absorbance of malondialdehyde (MDA) was measured at $532 \mathrm{~nm}$ by a Hitachi U-3200 spectrophotometer (Hitachi Ltd., Tokyo, Japan). The protein concentration of the $1300 \mathrm{~g}$ supernatant was measured using the BioRad Protein assay kit.

\subsection{Statistical Analysis}

All statistical analyses were performed using STATISTICA software (StatSoft, Tulsa, OK). Values were expressed as means \pm SD and checked by Kolmogorov-Smirnov Tests for normality before assessment of statistical significance. Differences were assessed by Student's $t$ test or one-way analysis of variance followed by post-hoc Tukey HSD test for parametric analysis and Mann-Whitney or Friedman ANOVA and Kendall Coeff of Concordance test for non-parametric analysis. The correlation was assessed by Pearson correlation analysis. P-values less than 0.05 were considered statistically significant.

\subsection{Declarations}

We followed the guidelines for experimental animal handling, and our study was approved by the Animal Care Committee of the Kyoritsu Women's University (\#15001). The experiment was conducted in accordance with the National Institutes of Health (NIH) guidelines.

\section{Results}

\subsection{Experiment I. Effects of Apple Juice on Cognitive Function}

To investigate the effects of fructose-rich foods on cognitive function, we examined whether apple juice affected memory function in a genetic rat model of human metabolic syndrome. Salt-sensitive Dahl rats develop salt-induced hypertension with decreased insulin sensitivity. To minimize insulin resistance due to fructose loading, we used lower concentrations of apple juice $\left(2.5^{\circ} \mathrm{Bx}\right)$ for 4 weeks than those required for induction of type 2 diabetes as reported previously [24].

No differences in the blood glucose concentration were observed between fructose-loaded and control rats, as shown in Table 1. In contrast, the systolic blood pressure at week 4 was $5.9 \%$ lower in the fructose group than in the control group $(\mathrm{p}<0.05)$. The memory function data are shown in Figure 2. The 
Table 1. Basal data on body weight, SBP and BS.

\begin{tabular}{cccccc}
\hline Group & \multicolumn{2}{c}{ Body weight (grams) } & \multicolumn{2}{c}{ SBP $(\mathrm{mmHg})$} & BS $(\mathrm{mg} / \mathrm{dL})$ \\
\hline Period & wk 0 & wk 4 & wk 0 & wk 4 & \\
\hline Control & $204.2 \pm 16.1$ & $311.4 \pm 16.7$ & $163.4 \pm 6.2$ & $153.5 \pm 6.8$ & $134.5 \pm 11.3$ \\
Fructose & $202.8 \pm 4.8$ & $316.4 \pm 16.5$ & $155.8 \pm 8.1$ & $146.1 \pm 3.2$ & $129.7 \pm 10.8$ \\
Apple juice & $204.2 \pm 11.3$ & $300.0 \pm 20.0$ & $159.1 \pm 6.4$ & $144.7 \pm 9.7^{*}$ & $134.0 \pm 15.5$ \\
\hline
\end{tabular}

Control, control group given water; Fructose, fructose group given $5 \%$ fructose solution; apple juice, rats given apple juice; SBP, systolic blood pressure; BS, 12-hr fasting blood glucose concentrations. Values are expressed as means $\pm \mathrm{SD}$. Differences are analyzed by Student's $\mathrm{t}$ test. ${ }^{*} \mathrm{p}<0.05$ vs control rats at wk 4 .

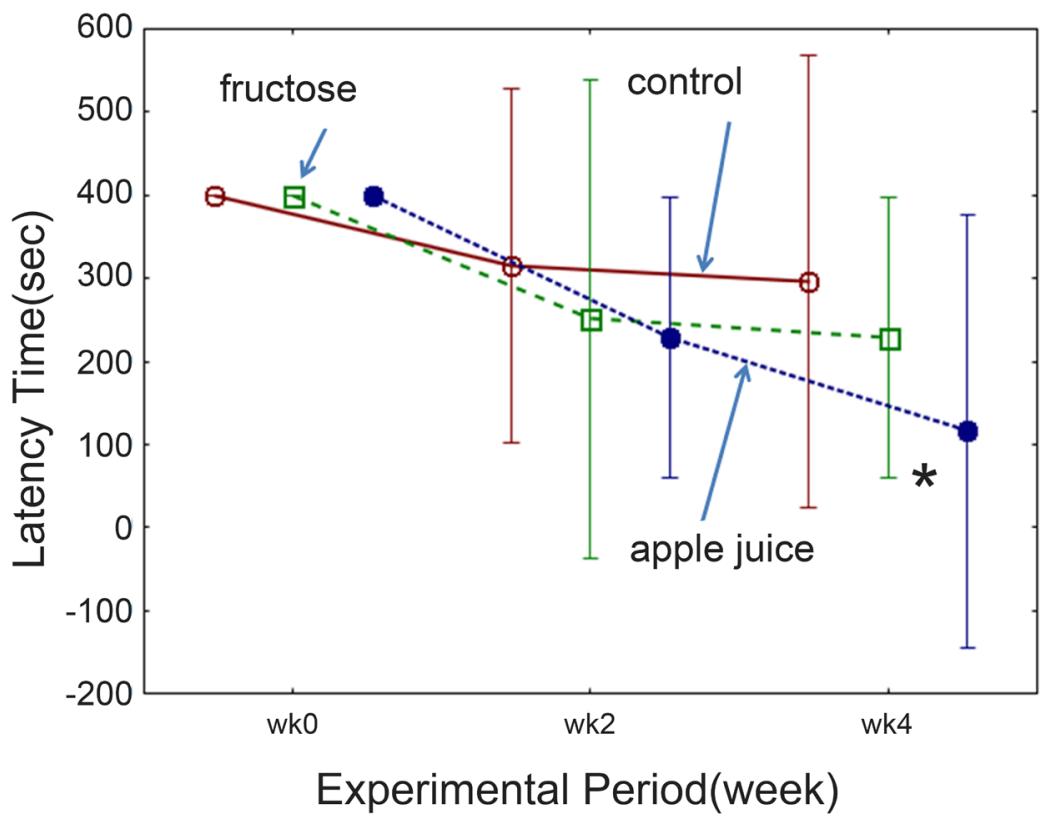

Figure 2. The effects of apple juice on memory function. Wk 0, prior to loading the solutions; wk 2, at week 2; wk 4, at the end of study; open circle, control rats; open square, rats given fructose solutions; and closed circle, the rats given apple juice. The differences were assessed by Mann-Whitney U test. ${ }^{*} \mathrm{p}<0.05$ vs control rats.

latency time was significantly decreased (by 60\%) in rats given apple juice compared with that in control rats $(\mathrm{p}<0.05)$.

\subsection{Experiment II. Effects of Long-Term Intake of Monosaccharides on Cognitive Function}

Based on the data in Exp I, we investigated the role of oxidative stress in cognitive impairment in rats given fruit juice solution.

Although the mean BW increased in an age-dependent manner, no significant differences were found among the experimental groups either at day 28 or day 56 (Figure 3). Moreover, no significant differences were observed in BW gain among the four groups.

The blood glucose concentrations are shown in Figure 4. The glucose concentration in the fructose group was slightly higher than those in the other 


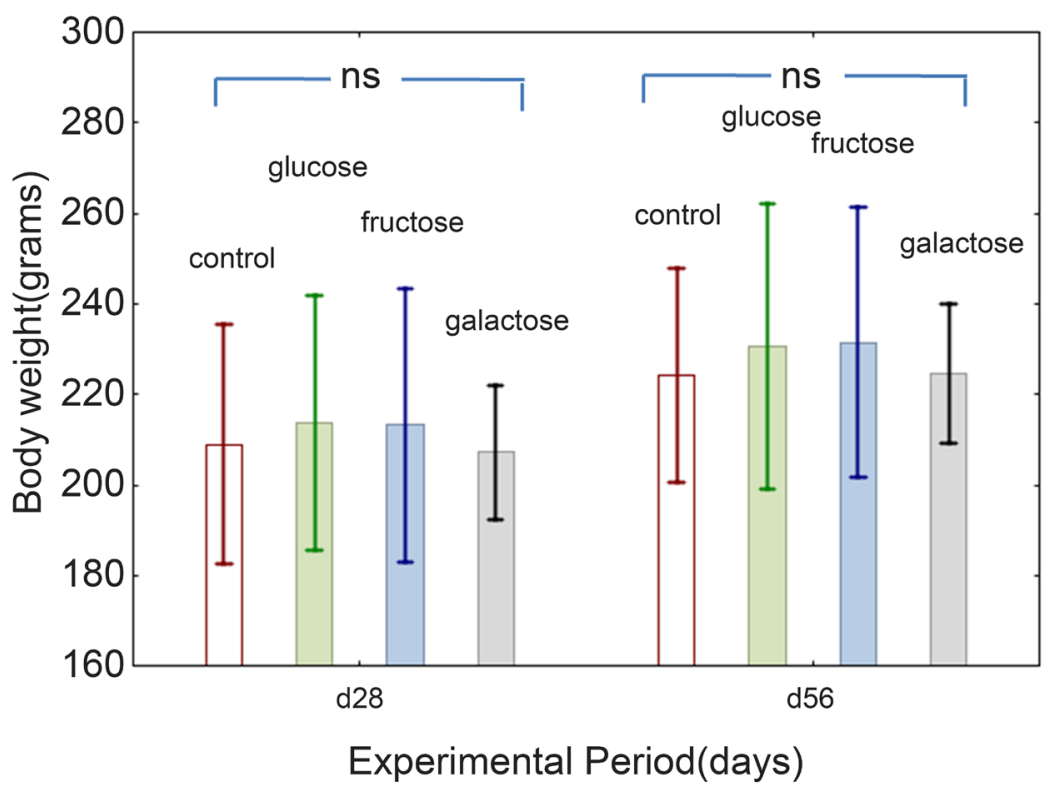

Figure 3. Body weight during the experiment. Body weights at day 28 are shown in the left columns and those at day 56 in the right columns. There were no differences in body weights among the experimental groups. The differences between the groups were assessed by Mann-Whitney U test.

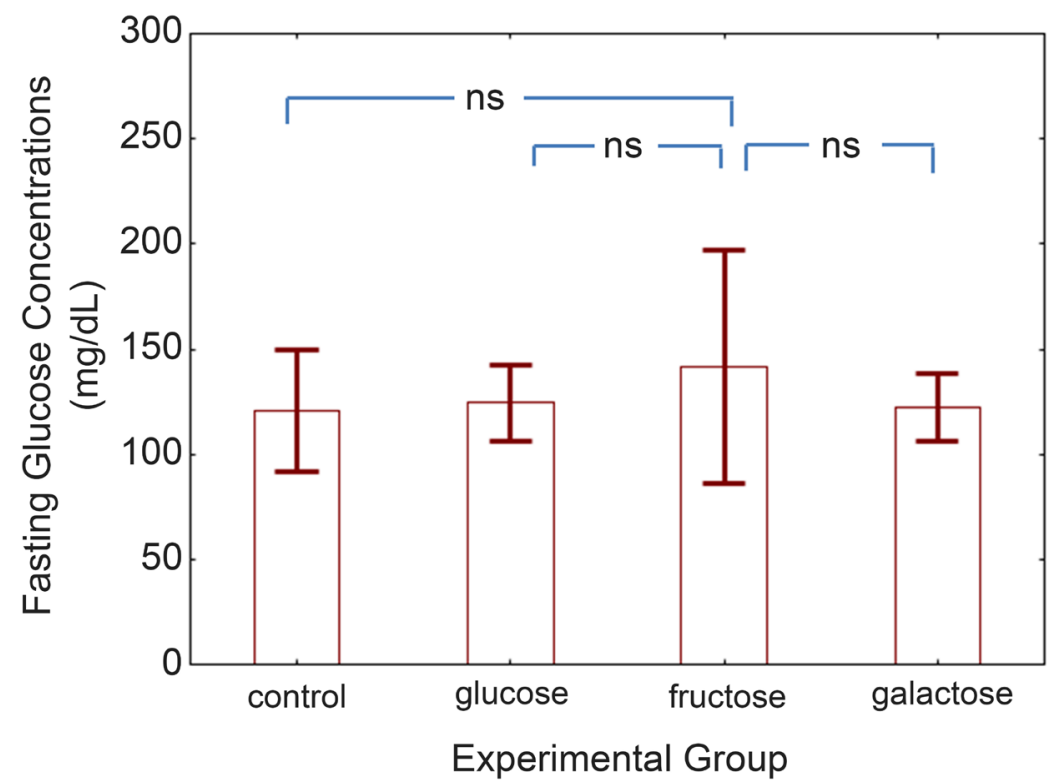

Figure 4. Blood glucose concentrations. Blood glucose concentrations after 12-hr fasting. Differences were assessed by Mann-Whitney U test. There were no group differences.

groups; however, the difference was not statistically significant (Mann-Whitney $\mathrm{U}$ test; $\mathrm{p}=0.118$ vs. control, $\mathrm{p}=0.443$ vs. glucose, and $\mathrm{p}=0.125$ vs. galactose).

The effects of monosaccharides on the response latency in the step-through passive avoidance task, a useful index of learning ability, are shown in Figure 5. Memory function was well preserved in the control and glucose groups throughout 


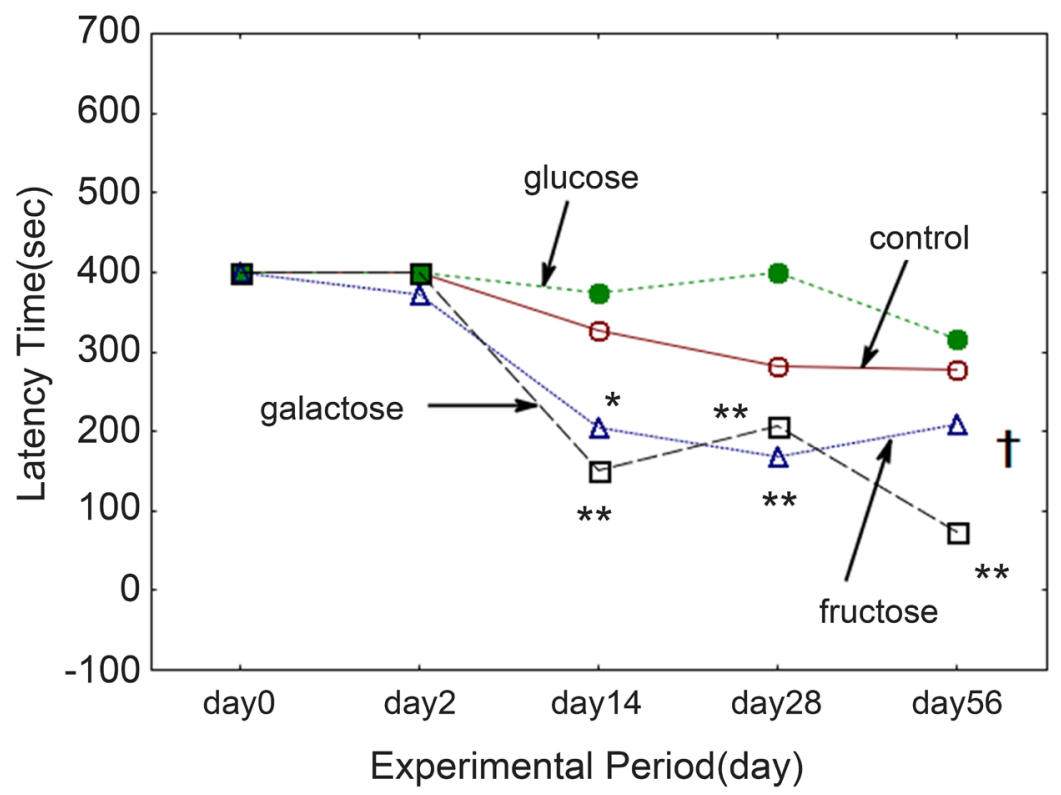

Figure 5. The effects of monosaccharides on memory function. Open circles, control; close circles, glucose group; open triangle, fructose group; and open square, galactose. Mean values ( $n=7$ for each) were demonstrated and SD values were not shown. The group differences during the experiment were assessed by Friedman ANOVA and Kendall Coeff of Concordance test; fructose vs control, $p<0.05$, fructose vs glucose, $p<0.001$ vs glucose, and galactose vs glucose, $\mathrm{p}<0.001$. The differences at each day were assessed by Mann-Whitney $\mathrm{U}$ test. ${ }^{\dagger} \mathrm{p}<0.1,{ }^{\star} \mathrm{p}<0.05,{ }^{* *} \mathrm{p}<0.001$ and ${ }^{\star * *} \mathrm{p}<0.0001$ vs glucose.

the experiment. In contrast, memory in the fructose group declined at days 14 , 28 , and 56 compared with that in the control and glucose groups. The group differences were significant (Friedman ANOVA test; $\mathrm{p}<0.05$ for fructose vs. control, $\mathrm{p}<0.001$ for fructose vs. glucose). The galactose group also exhibited lower memory function compared with the glucose group.

We measured cerebral MDA concentrations to assess oxidative stress in the brain. As shown in Figure 6(a), the brain MDA content tended to be higher in the fructose group than in the other experimental groups, the difference being significant with the glucose group $(\mathrm{p}<0.05)$. Moreover, the latency time significantly correlated with the brain MDA concentration $(r=-0.46, p<0.05)$ as shown in Figure 6(b).

\section{Discussion}

In this study, we demonstrated that, similar to fructose consumption, drinking diluted apple juice was associated with impaired memory. We reported that $15 \%$ fructose loading for 10 weeks causes apparent type-2 diabetes in rats. In this study, to reduce the influence of glucose metabolism, we used $2.5^{\circ} \mathrm{Bx}$ juice, which corresponded to lower dosage compared to that used for fructose loading in our previous study [24].

In this setting, no effect on the blood sugar concentration was observed; however, apple juice consumption was associated with impaired memory function 


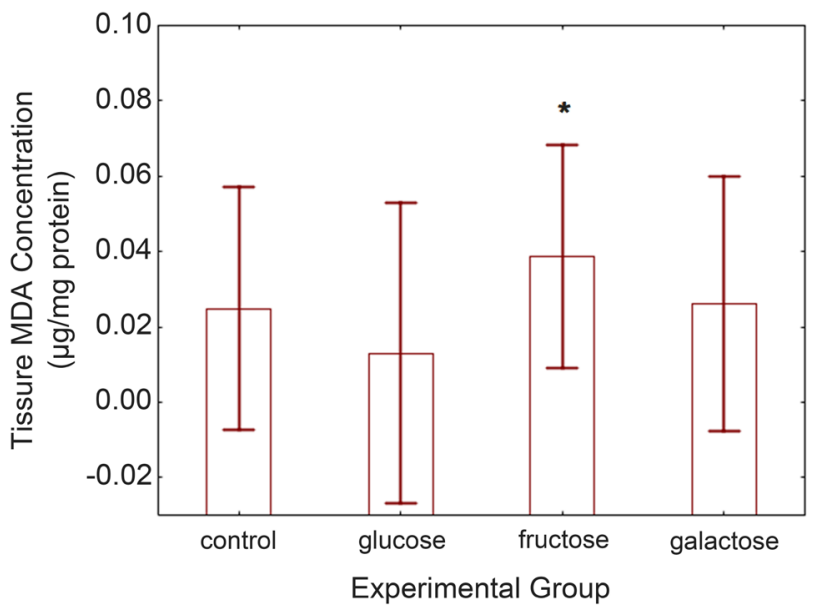

(a)

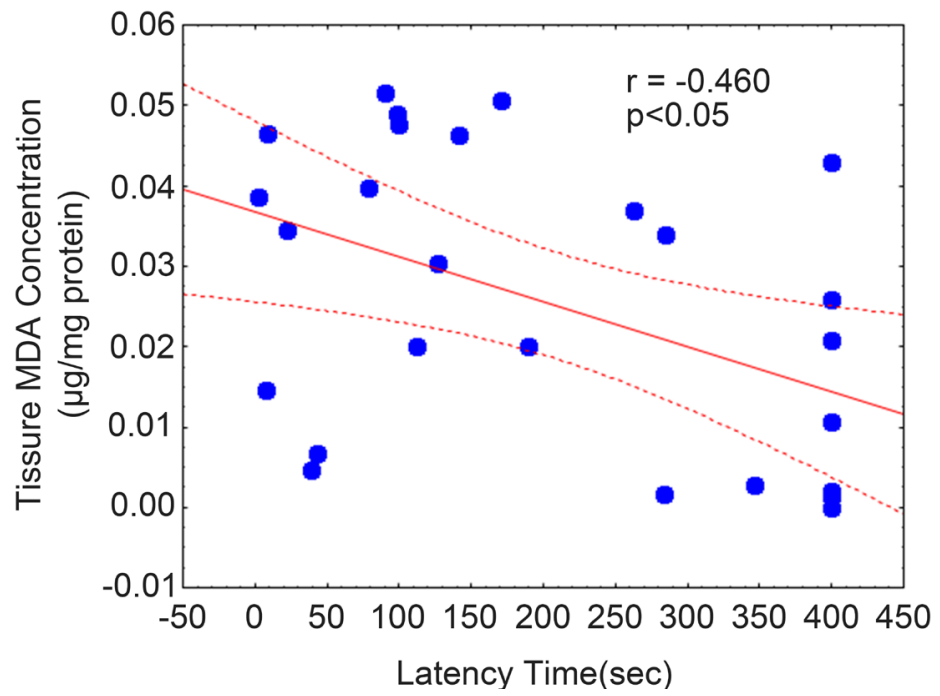

(b)

Figure 6. Oxygen stress in the brain. Oxygen stress measured as MDA concentrations in the brain was demonstrated in graph (a). The differences were assessed by Mann-Whitney U test. ${ }^{*} \mathrm{p}<0.05$ vs glucose. In graph (b), Pearson's correlation between MDA concentrations and memory function as assessed as latency time was demonstrated.

and a decreased systolic blood pressure compared with those of control rats. The latter result indicates that such low concentrations of apple juice do influence the rat cardiovascular system. However, no correlation was found between blood pressure levels and memory function $(\mathrm{r}=-0.064, \mathrm{~ns})$, suggesting that the memory impairment in rats given apple juice was not caused by the blood pressure reduction. Each $100 \mathrm{~mL}$ of $2.5^{\circ} \mathrm{Bx}$ apple juice contained $12.8 \mathrm{mg}$ of $\mathrm{K}^{+}$, translating into a daily intake of $42-62 \mathrm{mg} / \mathrm{kg} \mathrm{BW}$ based on the animals weighing 200 $300 \mathrm{~g}$ and consuming approximately $100 \mathrm{~mL}$ of juice a day. This intake is equivalent to $2.1-3.1 \mathrm{~g} / 50 \mathrm{~kg} \mathrm{BW}$ in humans, which is enough to reduce the blood pressure. 
We reported previously that long-term intake of $15 \%(\mathrm{w} / \mathrm{v})$ fructose solution caused type 2 diabetes in Wistar rats [24]. In these rats, increased blood glucose concentrations were associated with elevated blood insulin concentrations, thereby increasing the homeostasis model assessment of insulin resistance (HOMA-IR). The association of fructose loading with the onset of insulin resistance is in agreement with reports from other laboratories [2] [3] [4] [5] [6]. Interestingly, evidence is emerging that overt type 2 diabetes increases the risk of cognitive impairments [9]-[14]. Long-term hyperglycemia injures vascular walls and leads to a decrease in the cerebral blood flow. In addition, both insufficient glucose utilization and hyperinsulinemia impair cerebral cells responsible for memory function. Hyperinsulinemia due to insulin resistance down regulates insulin receptor expression and consequently accelerates the processes of neural aging and neurodegeneration.

Type 2 diabetes induced by long-term fructose intake has been reported to be associated with a decline in cognitive function [10] [11] [12] [13]. However, it is not clear whether the memory impairment is caused by diabetes or the excessive intake of fructose. In fact, evidence exists that fructose is cytotoxic through reduction-oxidation reactions [15] [16]. An important finding of the present study is that memory impairment, as assessed by the passive avoidance test, precedes overt type 2 diabetes: blood glucose concentrations did not increase following fructose loading in our experiments. These results show that the memory function deficits observed in fructose-fed rats are unlikely to be mediated by impaired glucose metabolism.

Takechi, et al. reported that fructose loading disrupted the BBB and increased its permeability in mice prior to cognitive function impairment [15]. The BBB is a target of oxidative stress. Moreover, it is reported that oxidative stress increases blood-brain barrier permeability and induces alterations in occludin during hypoxia-reoxygenation [25]. Indeed, we demonstrated that oxidative stress in rats given fructose was higher than in those fed glucose. Moreover, the stress level was negatively correlated with memory function. These results strongly suggest that memory dysfunction in fructose-fed rats was mediated by increased oxidative stress and $\mathrm{BBB}$ disruption. Thus, insulin resistance may induce cognitive dysfunction before glucose metabolism is impaired.

Galactose loading was associated with cognitive impairment; however, oxidative stress was not increased in the brain. Cui et al. reported that long-term administration of D-galactose in s.c. (100 mg/kg) for 7 weeks causes neurodegeneration with oxidative stress in serum [26]. However, memory impairment due to galactose loading is reportedly associated with neurodegeneration, decreased immune responses, advanced glycation end product (AGE) formation and gene transcriptional changes. Mechanism of memory impairment may be different between these monosaccharides.

We examined glucose, fructose and galactose in this study because these are the main monosaccharides in daily life. Some of monosaccharides interest us for 
health benefits on glucose metabolism and this is our next step to investigate.

\section{Conclusion}

We have demonstrated that long-term fructose intake is associated with impaired memory function and increased oxidative stress in the rat brain. Memory was also impaired in rats given apple juice rich in fructose, without overt hyperglycemia. These results suggest that fructose-intake-associated memory impairment is not related to insulin resistance but, rather, is caused by fructose loading per se. Fructose intake may need to be reduced to the minimum level required for healthy living.

\section{Funding Statement}

The authors acknowledge Grants-in-Aid from the Center for Interdisciplinary Studies of Science and Culture, Kyoritsu Women's University \& Junior College.

\section{Data Availability Statement}

The experimental data used to support the findings of this study are available from the corresponding author upon request.

\section{Conflicts of Interest}

The authors report no conflict of interests regarding the publication of this paper.

The authors alone are responsible for the content and writing of the paper.

\section{References}

[1] Ministry of Health, Labor and Welfare. The Second Term of National Health Promotion Movement in the Twenty First Century. http://www.mhlw.go.jp/seisakunitsuite/bunya/kenkou_iryou/kenkou/kenkounippo n21/en/eiyouchousa/kekka_todoufuken.html

[2] de Moura, R.F., Ribeiro, C., de Oliveira, J.A., Stevanato, E. and de Mello, M.A. (2009) Metabolic Syndrome Signs in Wistar Rats Submitted to Different High-Fructose Ingestion Protocols. British Journal of Nutrition, 101, 1178-1184. https://doi.org/10.1017/S0007114508066774

[3] Havel, P.J. (2005) Dietary Fructose: Implications for Dysregulation of Energy Homeostasis and Lipid/Carbohydrate Metabolism. Nutrition Reviews, 63, 133-157. https://doi.org/10.1111/j.1753-4887.2005.tb00132.x

[4] Dupas, J., Goanvec, C., Feray, A., Guernec, A., Alain, C., Guerrero, F. and Mansourati, J. (2016) Progressive Induction of Type 2 Diabetes: Effects of a Reality-Like Fructose Enriched Diet in Young Wistar Rats. PLOS One, 11, e0146821. https://doi.org/10.1371/journal.pone.0146821

[5] Elliott, S.S., Keim, N.L., Stern, J.S., Teff, K. and Havel, P.J. (2002) Fructose, Weight Gain, and the Insulin Resistance Syndrome1-3. The American Journal of Clinical Nutrition, 76, 911-922. https://doi.org/10.1093/ajcn/76.5.911

[6] Kelishadi, R., Mansourian, M. and Heidari-Beni, M. (2014) Association of Fructose Consumption and Components of Metabolic Syndrome in Human Studies: A Sys- 
tematic Review and Meta-Analysis. Nutrition, 30, 503-510. https://doi.org/10.1016/j.nut.2013.08.014

[7] Ministry of Agriculture, Forestry and Fisheries. http://www.maff.go.jp/kyusyu/seiryuu/yasaikudamono/pdf/200gundo 1.pdf

[8] Sun, S.Z., Anderson, H.G., Flickinger, B.D., Williamson-Hughes, P.S. and Empie, M.W. (2011) Fructose and Non-fructose Sugar Intakes in the US Population and Their Associations with Indicators of Metabolic Syndrome. Food and Chemical Toxicology, 49, 2875-2882. https://doi.org/10.1016/j.fct.2011.07.068

[9] Saedi, E., Gheini, M.R., Faiz, F. and Arami, M.A. (2016) Diabetes Mellitus and Cognitive Impairments. World Journal of Diabetes, 7, 412-422.

https://doi.org/10.4239/wjd.v7.i17.412

[10] Kravitz, E., Schmeidler, J. and Schnaider, B.M. (2013) Type 2 Diabetes and Cognitive Compromise: Potential Roles of Diabetes-Related Therapies. Endocrinology Metabolism Clinics of North America, 42, 489-501. https://doi.org/10.1016/j.ecl.2013.05.009

[11] Biessels, G.J., Strachan, M.W., Visseren, F.L., Kappelle, L.J. and Whitmer, R.A. (2014) Dementia and Cognitive Decline in Type 2 Diabetes and Prediabetic Stages: Towards Targeted Interventions. The Lancet Diabetes \& Endocrinology, 2, 246-255. https://doi.org/10.1016/S2213-8587(13)70088-3

[12] Janson, J., Laedtke, T., Parisi, J.E., O’Brien, P., Petersen, R.C. and Butler, P.C. (2004) Increased Risk of Type 2 Diabetes in Alzheimer Disease. Diabetes, 53, 474-481. https://doi.org/10.2337/diabetes.53.2.474

[13] Ravona-Springer, R., Luo, X., Schmeidler, J., Wysocki, M., Lesser, G., Rapp, M., Dahlman, K., Grossman, H., Haroutunian, V. and Schnaider, B.M. (2010) Diabetes Is Associated with Increased Rate of Cognitive Decline in Questionably Demented Elderly. Dementia and Geriatric Cognitive Disorders, 29, 68-74. https://doi.org/10.1159/000265552

[14] Lakhan, S.E. and Kirchgessner, A. (2013) The Emerging Role of Dietary Fructose in Obesity and Cognitive Decline. Nutrition Journal, 12, 114.

https://doi.org/10.1186/1475-2891-12-114

[15] Takechi. R., Lam, V., Brook, E., Giles, C. and Fimognari, N. (2017) Blood-Brain Barrier Dysfunction Precedes Cognitive Decline and Neurodegeneration in Diabetic Insulin Resistant Mouse Model: An Implication for Causal Link. Front Aging Neurosci, 1 December 2017. https://doi.org/10.3389/fnagi.2017.00399

[16] Francisqueti, F.V., Santos, K.C., Ferron, A.J.T., Lo, A.T.C., Minatel, I.O., Campos, D.H.S., Ferreira, A.L.A. and Corrêa, C.R. (2016) Fructose: Toxic Effect on Cardiorenal Risk Factors and Redox State. SAGE Open Medicine, 4, Article ID: 2050312116684294.

[17] Hirawa, N., Uehara, Y., Kawabata, Y., Oshima, N., Ono, H., Nagata, T., Gomi, T., Ikeda, T., Goto, A., Yagi, S. and Omata, M. (1994) Mechanistic Analysis of Renal Protection by Angiotensin Converting Enzyme Inhibitor in Dahl Salt-Sensitive Rats. Journal of Hypertension, 12, 909-918. https://doi.org/10.1097/00004872-199408000-00008

[18] Hirawa, N., Uehara, Y., Kawabata, Y., Numabe, A., Oshima, N., Ono, H., Gomi, T., Ikeda, T., Yagi, S., Toyo-Oka, T. and Omata, M. (1995) Subpressor Dose of Angiotensin II Increases Susceptibility to the Haemodynamic Injury of Blood Pressure in Dahl Salt-Sensitive Rats. Journal of Hypertension, 13, 81-90. https://doi.org/10.1097/00004872-199501000-00013

[19] Hirawa, N., Uehara, Y., Kawabata, Y., Numabe, A., Gomi, T., Ikeda, T., Suzuki, T., 
Goto, A., Toyo-oka, T. and Omata, M. (1999) Long-Term Inhibition of Renin-Angiotensin System Sustains Memory Function in Aged Dahl Rats. Hypertension, 34, 496-502. https://doi.org/10.1161/01.HYP.34.3.496

[20] Braszko, J.J. and Wisniewski, K. (1988) Effects of Angiotensin II and Saralasin on Motor Activity and the Passive Avoidance Behavior of Rats. Peptides, 9, 475-479. https://doi.org/10.1016/0196-9781(88)90150-7

[21] Tadokoro, S., Kuribara, H. and Hayashi, H. (1987) Methodological Problems on Learning and Memory Tests in Rodents from View Points of Behavioral Toxicology. In: Fujii, T. and Adams, P.M., Eds., Functional Teratogenesis. Functional Effects on the Offspring after Parenteral Drug Exposure, Teikyo University Press, Tokyo, 53-70.

[22] Uehara, Y., Numabe, A., Hirawa, N., Kawabata, Y., Iwai, J., Ono, H., Matsuoka, H., Takabatake, Y., Yagi, S. and Sugimoto, T. (1991) Antihypertensive Effects of Cicletanine and Renal Protection in Dahl Salt-Sensitive Rats. Journal of Hypertension, 9 , 719-728. https://doi.org/10.1097/00004872-199108000-00005

[23] Uehara, Y., Kawabata, Y., Shirahase, H., Wada, K., Morishita, S., Numabe, A., Iwai, J., Matsuoka, H. and Sugimoto, T. (1992) Radical Scavengers of Indapamide and Renal Protection in Dahl Salt Sensitive Rats. Hypertension Research, 15, 17-26. https://doi.org/10.1291/hypres.15.17

[24] Yamakawa, S., Oikawa, A., Ohta, M., Kanemaki, A., Mitsuhashi, N. and Uehara, Y. (2018) Wasabi Increases Insulin Sensitivity and Attenuates Glomerular Hyperfiltration and Proteinuria in Insulin-Resistant Diabetes of Fructose-Fed Wistar Rats. Proceedings of Kyoritsu Women's University Kiyo, 64, 63-71.

[25] Lochhead, J.J., McCaffrey, G., Quigley, C.E., Finch, J., DeMarco, K.M., Nametz, N. and Davis, T.P. (2010) Oxidative Stress Increases Blood-Brain Barrier Permeability and Induces Alterations in Occludin during Hypoxia-Reoxygenation. Journal of Cerebral Blood Flow \& Metabolism, 30, 1625-1636. https://doi.org/10.1038/jcbfm.2010.29

[26] Cui, X., Zuo, P., Zhang, Q., Li, X., Hu, Y., Long, J., Packer, L. and Jiankang, L.J. (2006) Chronic Systemic D-Galactose Exposure Induces Memory Loss, Neurodegeneration, and Oxidative Damage in Mice: Protective Effects of R-a-lipoic Acid. Journal of Neuroscience Research, 84, 647-654. https://doi.org/10.1002/jnr.20899

\section{Abbreviations}

AGE: Advanced glycation endproduct Dahl S rats: Dahl salt-sensitive rats ANOVA: One-way factorial analysis of variance BBB: Blood brain barrier

BW: Body weight

${ }^{\circ} \mathrm{Bx}$ : percentage Brix

D-PBS: Dulbecco's phosphate buffered solution

HOMA-IR: Homeostasis model assessment of insulin resistance

MDA: Malondialdehyde 